BRAVILLIAN JOURNAL

www.bjournal.com.br
ISSN 0100-879X

Volume 43 (4) 268-380 April 2011

BIOMEDICAL SCIENCES

AND

CLINICAL INVESTIGATION

Braz J Med Biol Res, April 2011, Volume 44(4) 268-275

doi: 10.1590/S0100-879X2011007500031

The fallacy of racial pharmacogenomics

S.D.J. Pena

The Brazilian Journal of Medical and Biological Research is partially financed by

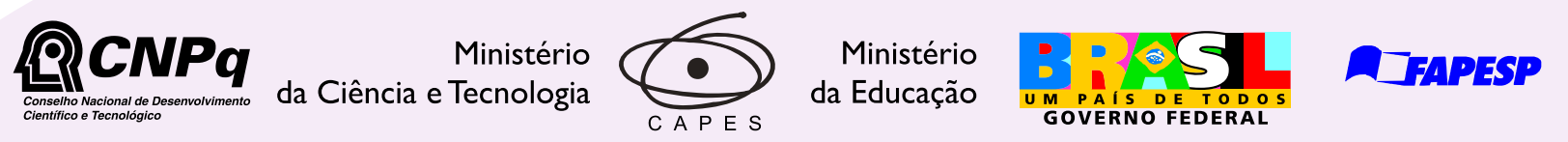

Institutional Sponsors
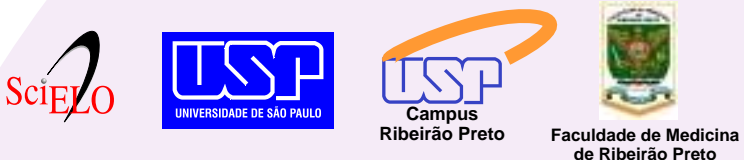

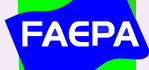

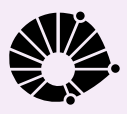

Ф SHIMADZU

GE Healthcare
Hotsite of proteomics metabolomics developped by:

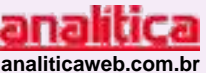

Thermo SCIENTIFIC 


\title{
The fallacy of racial pharmacogenomics
}

\author{
S.D.J. Pena ${ }^{1,2}$ \\ ${ }^{1}$ Departamento de Bioquímica e Imunologia, Universidade Federal de Minas Gerais, \\ Belo Horizonte, MG, Brasil \\ ${ }^{2}$ GENE - Núcleo de Genética Médica, Belo Horizonte, MG, Brasil
}

\begin{abstract}
Personalized pharmacogenomics aims to use individual genotypes to direct medical treatment. Unfortunately, the loci relevant for the pharmacokinetics and especially the pharmacodynamics of most drugs are still unknown. Moreover, we still do not understand the role that individual genotypes play in modulating the pathogenesis, the clinical course and the susceptibility to drugs of human diseases which, although appearing homogeneous on the surface, may vary from patient to patient. To try to deal with this situation, it has been proposed to use interpopulational variability as a reference for drug development and prescription, leading to the development of "race-targeted drugs". Given the present limitations of genomic knowledge and of the tools needed to fully implement it today, some investigators have proposed to use racial criteria as a palliative measure until personalized pharmacogenomics is fully developed. This was the rationale for the FDA approval of BiDil for treatment of heart failure in African Americans. I will evaluate the efficacy and safety of racial pharmacogenomics here and conclude that it fails on both counts. Next I shall review the perspectives and the predicted rate of development of clinical genomic studies. The conclusion is that "next-generation" genomic sequencing is advancing at a tremendous rate and that true personalized pharmacogenomics, based on individual genotyping, should soon become a clinical reality.
\end{abstract}

Key words: DNA; Genetic polymorphisms; Human genomic variability; Race; Pharmacogenomics

\section{Introduction}

The American astrophysicist Neil deGrasse Tyson defined the "perimeter of ignorance" as the boundary where scientists face a choice: continue the quest for knowledge or invoke a deity or other supernatural forces (1). He used as an example no less than Isaac Newton himself, whose law of gravity enabled calculation of the force of attraction between any two objects. When computing the orbits of the planets around the sun, Newton feared that the mutual attraction between them would render the solar system unstable. He then concluded that God occasionally stepped in to make things right. A century later, the French astronomer Pierre-Simon de Laplace created a new mathematical tool called perturbation theory and used it to demonstrate that the solar system is in fact stable over periods of time much longer than Newton could predict. Laplacian science, therefore, no longer needed to postulate the interference of supernatural forces to explain astronomical facts.

Newton's appeal to God, however unnecessary, may at first sight appear as a humble attitude of a great man. However, Tyson demonstrates that, on the contrary, it represented presumptuousness on his part: if his mathematics was not good enough to explain the phenomenon, then the problem was too complicated for any other human mind to figure out, then or anytime in the future. By "embracing ignorance" Newton's attitude negatively infused a temporary stage of incomplete knowledge with a false permanency, running counter to the philosophy of open-mindedness and discovery that characterizes Science.

Pharmacogenetics and pharmacogenomics are likewise in a dilemma right at the edge of the perimeter of ignorance.

We know that the safety and efficacy of drug treatment differs in humans, depending on genes that influence pharmacokinetics and pharmacodynamics. Such variability may occur at two different genetic levels. The first is interpersonal, i.e., the diversity that distinguishes one person from all the others in the same population and which is intimately connected to individual identity, uniqueness. The second is the interpopulational level, collective, the diversity that distinguishes different human groups, including the so-called "races". (Since there is consensus among anthropologists and human geneticists that, from a biological standpoint,

Correspondence: S.D.J. Pena, GENE - Núcleo de Genética Médica, Av. Afonso Pena, 3111/9, 30130-909 Belo Horizonte, MG, Brasil. Fax: +55-31-3227-3792. E-mail: spena@gene.com.br

Received January 11, 2011. Accepted February 14, 2011. Available online March 11, 2011. Published April 11, 2011. 
human races do not exist (2), I believe that the word "race" should always be mentioned between quotation marks. However, the excess of quotation marks truncates the text and will be omitted in this article for this reason only.)

For pharmacological agents with well-characterized metabolism and mechanisms of action, it would in theory be possible to assess the genetic variation in pertinent loci and to use patient genotypes to direct medical treatment the dream of providing the right drug, in the right dosage, to the right patient. This is the theoretical underpinning of personalized pharmacogenomics. Unfortunately, the loci relevant for the pharmacokinetics and especially the pharmacodynamics of most drugs are still unknown. Moreover, we still lack comprehension of the role that individual genotypes play in modulating the pathogenesis, the clinical course and the drug susceptibility of human diseases which, although appearing homogeneous on the surface, may vary from patient to patient.

To try to deal with this situation it has been proposed to use interpopulational variability as a reference for drug development and prescription, leading to the development of "race-targeted drugs", as exemplified by the case of BiDil for treatment of heart failure in African Americans. The rationale for such strategy is that, since we still lack the pharmacogenomic knowledge necessary to implement true personalized treatment, we make do by using the race or the ethnic-geographic affiliation of a given patient as the replacement of the germane individual genotyping at critical loci.

Therein lies the fallacy of racial pharmacogenomics being predicated on the idea that individual genotyping will be impossible to achieve in the near future, it "embraces ignorance". Moreover, it often does so under false premises. For instance, in the FDA news release entitled "FDA Approves BiDil Heart Failure Drug for Black Patients" (3) it is stated that this represents "a step toward the promise of personalized medicine". But racial medicine is group medicine - most definitely it is not personalized medicine.

Jones and Goodman (4) report that at a meeting just preceding the approval of BiDil, Steve Nissen, chair of the FDA Cardiovascular and Renal Drugs Advisory Committee, made the following pronouncement: "...we are moving toward the era of genomic-based medicine. There is no question that in 10 or 15 years it is going to happen. I know it has been predicted for a long time and has not happened yet but is going to happen, trust me. [...] So what we are doing is we are using self-identified race as a surrogate for genomic-based medicine."

Thus, the big question is: should we wait for the inevitable development of proper personalized pharmacogenomics science or should we adopt obviously palliative racial measures as announced by the FDA? Common precaution indicates that, before we make a decision on this dilemma, we should evaluate the efficacy and safety of racial pharmacogenomics, in the same way that we evaluate a new pharmacological agent. I start this article by making such analysis and presenting evidence and arguments that reveal the failure of racial pharmacogenomics on both efficacy and safety counts. I will then conclude with an assessment regarding the predicted rate of development of personalized pharmacogenomics. The good news is that it is advancing at almost warp speed with "next-generation sequencing" and should soon become a clinical reality.

\section{The efficacy and safety of racial pharmacogenomics}

There is consensus among anthropologists and human geneticists that, from a biological standpoint, human races are not biological entities, but social constructs $(2,5,6)$.

However, a retrospective review of clinical trial protocols and product labeling for 185 new molecular entities approved between 1995 and 1999 showed that labeling for $45 \%(84 / 185)$ of the products contained some statement about race and that in $8 \%(15 / 185)$ of cases race-related differences were described (7). Moreover, in 2005 the FDA approved BiDil avowedly "for the treatment of heart failure in self-identified black patients" (3). Thus, from a pharmacological perspective, human races are still being treated as "real" biological categories.

A peculiar aspect of the literature about "race" is that there does not appear to be a consensus about its exact meaning, which is often subjected to ideological manipulation (8). We can actually identify different ways in which the word "race" is used. The first is phenotypic, generally associated with iconic physical characteristics, more especially skin color, such as Blacks, Whites, etc. "Race" can also assume the meaning of "geographic ancestry", such as Africans, Asians, etc. But the rotary machine gun of race spares no one. "Race" has also been used, when politically convenient, to denote groups, which have in common religion and/or culture, such as the case of the Jewish people, who are not a "race" in either the phenotypic or geographical sense, and yet were the victims of the "racially" perpetrated Nazi holocaust. "Race" can finally be applied even to persons with very vague elements in common, such as the bizarre American category of "Hispanics", which covers all Mexicans, Central Americans and South Americans, including Portuguese-speaking Brazilians. I wish to briefly discuss the first two meanings of race separately.

\section{Races as phenotypes}

In the FDA approval of Bidil (3), "Blacks" were singled out as a racial category because of their skin pigmentation. However, we know that skin color is controlled by a handful of genes (9) that are a minuscule proportion of the more than 20,000 genes contained in the human genome. Thus, a person's color, or any other of the iconic racial characteristic, does not have anything to do with his intelligence, 
personality, abilities, blood pressure, or prostate health.

One of the biggest mistakes of racial pharmacogenomics is to assume that Africa is homogeneous and all "Blacks" are the same. On the contrary, the variability within Africa is larger than the genetic variability between Africa and Europe (10). One example of this, highlighted by $\mathrm{Ng}$ et al. (11), is that of CYP2D6, involved in the metabolism of several drugs. The CYP2D6*17 allele, which is associated with lower enzyme activity, has a frequency of 0.9 in Ethiopia, 0.17 in Tanzania, and 0.34 in Zimbabwe. Obviously, the expectation of CYP2D6 activity of a Black person in America would depend on where in Africa his ancestors originated. Africa is still poorly studied from the genetic standpoint. However, considering the fact that humankind has spent two thirds of it evolutionary history in Africa (12) and also the tremendous variety of human geographical environments (forests, savannahs, deserts, mountains) on that vast continent, it is expected that allele frequencies at several pharmacogenomic loci should vary considerably in different regions.

Phenotypic and geographical racial classifications clearly purport to partition human genetic diversity on the basis of very different criteria. However, in spite of that, they are very often confused. Typical of this, and by no means an isolated example, is the case of a recent article of the International Warfarin Pharmacogenetics Consortium (13) that studied the predictive power of a genetic polymorphism of the gene VKORC1 (SNP rs9923231, also known as $-1639 \mathrm{G}>\mathrm{A}$ ) regarding the dose of the anti-coagulant warfarin for "4886 patients of known race", a sample composed of "1103 Asians, 670 blacks and 3113 white individuals". The authors thus used a disjoint racial classification that mixed phenotypic and geographical criteria.

There is also a tendency to confuse New World populations with their ancestors, which is often convenient, but certainly conceptually wrong. For instance, in the original "mitochondrial Eve" paper, the authors used African Americans as if they were Africans (14). Even the more recent and carefully planned HapMap study used groups of Utah
Mormons as if they were Europeans (15).

To demonstrate the danger involved in these practices, we retrieved from HapMap 3 (16) data aboutAfrican Yorubas from Ibadan, Nigeria (YRI - 113 individuals) and European Tuscans from Italy (TSI - 88 individuals), two populations that differed in continental origin. We also retrieved American Blacks from the Southwest USA (ASW - 48 individuals) and American Whites from Utah, USA (CEU - 112 individuals). In all groups we ascertained genotypes at the rs9923231 SNP, the same that, according to Limdi et al. (13), explained the greatest variance in warfarin dose.

The frequencies of the C allele of SNP rs9923231 differed considerably between African Yorubans and European Tuscans (geographical groups), with a differential (ठ) of 0.455 (Table 1). On the other hand, the frequencies of American Blacks and Whites (phenotypic groups) were more similar, with a smaller differential $(\delta)$ of 0.299 . The analysis of molecular variance of the American color categories (ASW vs CEU) showed that $82.6 \%$ of the variation occurred within the populations and only $17.4 \%$ between them, while in the geographical contrast (YRI vs TSI) $54.2 \%$ of the variation occurred within the populations and $45.8 \%$ between them (Table 1). This example makes clear the hazards of equating color to geographical ancestry and interchangeably using terms such as White and European on one hand, and Black and African on the other, as is often done in racial pharmacogenomics.

The racial category "Black" was used by the FDA in the BiDil (3) approval as a proxy for a pharmacogenomic genotype. However, it is clear that skin color and other iconic racial characteristics are not adequately powerful parameters to choose a dosage schedule or a specific medicine.

\section{Races as geographical groups}

After World War II and the revelation of the racial atrocities of the Nazis, geneticists and anthropologists decided that it was high time to abandon the typological racial paradigms inherited from the 19th century "scientific racism"

Table 1. Analysis of single nucleotide polymorphism (SNP) rs9923231 in HapMap 3 (Ref. 16) populations.

\begin{tabular}{|c|c|c|c|c|c|c|}
\hline \multirow[t]{2}{*}{ HapMap population } & \multirow[t]{2}{*}{$\begin{array}{c}\text { No. of } \\
\text { individuals }\end{array}$} & \multicolumn{2}{|c|}{$\begin{array}{l}\text { SNP rs9923231 } \\
\text { C allele }\end{array}$} & \multirow[t]{2}{*}{$\begin{array}{l}\text { Allele frequency } \\
\text { differential }\end{array}$} & \multicolumn{2}{|c|}{$\begin{array}{c}\text { Analysis of molecular } \\
\text { variance }\end{array}$} \\
\hline & & Frequency & $\begin{array}{l}\text { 95\% confidence } \\
\text { limits }\end{array}$ & & $\begin{array}{l}\text { Among population } \\
\text { component }\end{array}$ & $\begin{array}{l}\text { Within population } \\
\text { component }\end{array}$ \\
\hline Western Africans (YRI) & 113 & 0.978 & 0.010 & \multirow{2}{*}{0.455} & \multirow{2}{*}{$45.8 \%$} & \multirow{2}{*}{$54.2 \%$} \\
\hline Western Europeans (TSI) & 88 & 0.523 & 0.038 & & & \\
\hline American Blacks (ASW) & 48 & 0.896 & 0.031 & \multirow{2}{*}{0.299} & \multirow{2}{*}{$17.4 \%$} & \multirow{2}{*}{$82.6 \%$} \\
\hline American Whites (CEU) & 112 & 0.597 & 0.033 & & & \\
\hline
\end{tabular}

African Yorubas from Ibadan, Nigeria (YRI) and European Tuscans from Italy (TSI) differ geographically, while American Blacks from Southwest USA (ASW) and American Whites from Utah, USA (CEU) differ in color phenotypes. 
movement and to adopt a model of division of humankind into populations. However, in spite of declarations to the contrary such as the one from UNESCO in 1950 (http:// unesdoc.unesco.org/images/0012/001282/128291eo.pdf, 1949), the concept of human races still survived, although in a new statistical plumage. The most accepted position at the time was that of Dunn and Dobzhansky (17) who defined races as geographically distinct human populations that differed in genetic variables.

It is curious to note that the idea of geographical continental human races dates all the way back to Johann Friedrich Blumenbach (1752-1840), who proposed in his book De Generis Humani Varietate Nativa the existence of five human races inhabiting different continents: Caucasians (Europe, Middle East, India, and North Africa), Mongoloids (East Asia), Ethiopians (Sub-Saharan Africa), Americans (Americas), and Malays (Oceania) (http://discovermagazine.com/1994/nov/thegeometerofrac441). Many elements of the classification of Blumenbach still persist, including the peculiar denomination "Caucasian".

As previously mentioned, the total genetic variation of human populations can be described on two levels: an interpersonal component (within-populations) and an interpopulational component (among-populations). Lewontin (5) was the first to quantitatively compare these components in an effort to evaluate the appropriateness of the division of humankind into distinct races. He compiled the literature on human genetic variation available in 1972, consisting of 17 genes, which comprised blood groups, transplantation antigens and polymorphisms of serum proteins and soluble enzymes. To a general surprise, he observed that $85.4 \%$ of the variance occurred within populations, $8.3 \%$ between different population of the same continent and only $6.3 \%$ between continents (i.e., the so-called "continental races"). On these bases, Lewontin concluded that the division of humankind into races had no genetic significance (5).

In 2002 a very influential study was published by Rosenberg et al. (18) using the same general geographical design, but utilizing for the first time the reference panel of DNA samples that had been collected by the Human Genome Diversity Program and stored at the Fondation Jean Dausset-Centre d'Études du Polymorphisme Humain in Paris. This HGDP-CEPH panel is a resource of cultured lymphoblastoid cell lines from 1050 individuals in 51 world populations distributed among Europe, the Middle East, North Africa, Central Asia, East Asia, Sub-Saharan Africa, Oceania, and America (http://www.cephb.fr/en/hgdp/diversity.php).

On genotyping a set of 377 autosomal microsatellite loci in all the samples, Rosenberg et al. (18) observed a total of 4199 alleles, $47 \%$ of which were present in all populations tested. Only $7 \%$ of the alleles were present on a single continent, which in the majority of cases was subSaharan Africa. These results were perfectly compatible with the origin of modern mankind in Africa and with a very small degree of genetic differentiation of human continental populations. Indeed, they found that the within-population variation among the individuals accounted for $93-95 \%$ of the total genetic variance, while the among-region variation represented only $3.6 \%$.

Next the authors decided to ascertain the capacity of the microsatellites to differentiate human groups. They used a Bayesian clustering algorithm that was successful in statistically partitioning human genomic variability into five geographical clusters: Sub-Saharan Africa, East Asia, Oceania, America and a fifth set composed of Europe, North Africa, the Middle East, and Central Asia.

Prima facie, one could recognize here a homology with the five human races defined over one hundred years ago by Blumenbach. Indeed, authors in both the scientific literature (e.g., Ref. 19,20) and the press (21) claimed that the study of Rosenberg et al. (18) had in fact reestablished the notion of human races on modern post-genomic grounds. However, such views are erroneous and cannot withstand close scrutiny. Several lines of evidence indicate methodological and interpretation problems and it is important to understand them to avoid wrong conclusions.

First of all, there is a problem with the populations sampled in the panel, which is not truly representative of worldwide genetic diversity. These were not DNA samples collected in a deliberate way to create a panel. Rather, they were initially obtained by independent groups for their own research and later grouped as an "international panel".

Secondly, even though the analysis using the clustering algorithm was undertaken without a priori population classification, the sampling strategy was clearly population-based. For instance, two of the five (40\%) Amerindian populations present, the Karitiana and the Surui, are genetically close. Both belong to the same Tupi linguistic group and both are from Rondônia, Brazil, separated by a distance of only 420 $\mathrm{km}$. Besides, most likely these samples contain several related individuals since they were collected from single Karitiana and Surui villages (22).

Also, the fact that only 127 samples from seven populations of Africa are available in the panel is grossly inadequate if we consider the vastness of the continent and the fact that it harbors the largest amount of human genetic diversity on the planet (10). The panel even lacks samples from northeast Africa, from where humans migrated 60,000 years ago to populate the rest of the world (12).

Such lack of representativity can create false discontinuities, maximizing the genetic variation between the continental groups and artificially creating quantum jumps of allele frequencies between them. The high geographical mobility of humans would lead us to expect gene frequency clines rather than geographical discontinuities. Indeed, Serre and Paabo (23) used simulation studies to show that if sampling had been done with individuals on a geographical grid, rather than being population-based, the clustering effect would be much diminished and the clinical structure 
of human diversity would be revealed.

Third, racial classifications have been based on the wrong typological idea that races were very different from each other and very internally homogeneous. That is not the picture that emerges from the data of Rosenberg et al. (18), which on the contrary show very heterogeneous clusters barely different from each other.

This becomes more evident when instead of focusing on continental groups one looks at the populations. Figure 1 shows an unrooted neighbor-joining tree of all the individuals in the HGDP-CEPH panel analyzed with 40 insertiondeletion polymorphisms (24). On the left panel of Figure 1 (A) the individuals are color coded according to which of five major geographic regions of the globe they originate from. Agood, although not perfect, aggregation of individuals can be observed. On the right panel $(B)$ individuals are color coded according to which of the 51 populations they come from. Now the impression of order disappears and the colors are shuffled. Similar trees can be obtained with Rosenberg's original data $(25,26)$. As pointed out by Weiss and Long (27), this clearly demonstrates that the way the data are interpreted is crucial.

The study by Rosenberg et al. (18) had a purely geographical structure. It treated the regions as if the enormous variation between the San and Yorubas in Sub-Saharan Africa on the one hand, and the small variation between Karitiana and Surui in South America on the other were equivalent. In other words, human evolutionary history, especially genealogical relationships between continental groups, was not taken into account.

Long et al. (26) showed that the adoption of such a model independent of human history has serious consequences, because: i) it underestimates diversity within African populations and overestimates diversity within European and Asia populations; ii) it underestimates diversity between African populations and overestimates diversity between European and Asian populations, and iii) it underestimates diversity between Africans and Asians, it underestimates diversity between Africans and Europeans, and it overestimates diversity between Europeans and Asians. Long et al. (26) obtained much better results when they tried to accommodate evolutionary history with models containing multiple levels of nesting and variable branch length. This is incompatible with a classification of human diversity into races, since the usual genetic and anthropological racial classifications place continental populations at the same level of classification. Thus, we conclude that the results of Rosenberg et al. (18) do not in fact support a racial model of human diversity.

Treating people, for instance, of the European population and African population, as separate categories for genetic studies tends to contribute to the public perception that the primary difference between these ways of defining populations is biological (28). This view confounds several issues and obscures the important fact that Europeans and Asians
A

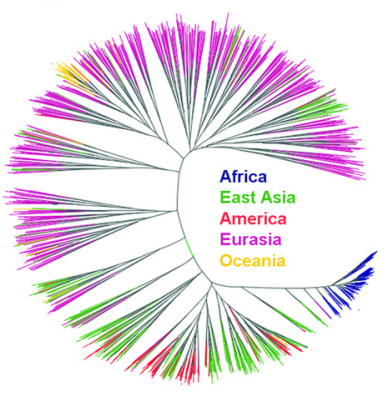

B

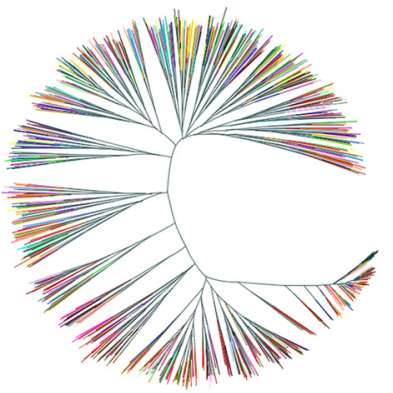

Figure 1. Neighbor-joining trees showing the genetic relationships of 1050 individuals from the HGDP-CEPH panel studied with a panel of 40 insertion-deletion autosomal polymorphisms (Ref. 24). This was inspired by an idea of Nievergelt et al. (Ref. 25). We used a distance matrix (Ref. 24) to construct the neighbor-joining tree using the neighbor module of the Phylip v.3.64 program (http://evolution.genetics.washington.edu/phylip.html). The branches were colored using the program HyperTree v.1.0.0 (http://www.kinase.com/tools/HyperTree.html) and the following procedure: in Panel $A$ the individuals are color coded according to which of five major geographic regions of the globe they originate from and in Panel $B$ individuals are color coded according to which of the 51 populations they come from.

are genealogically related to Africans, having evolved as an offshoot of the latter.

I propose that, rather than thinking about populations, ethnicities or races, we should focus on the unique genome of a particular individual, which is structured as a mosaic of polymorphic haplotypes with diverse genealogical histories (29). This shifts the emphasis from populations to persons. We should strive to see each individual as having a singular genome and a unique life history, rather than try to impose on $\mathrm{him} /$ her characteristics of a group or population. Under this model, ideas such as that of human races or "race-targeted drugs" become meaningless and vanish like smoke.

\section{The safety of racial pharmacogenomics}

The adoption of racial pharmacogenomics by the FDA has serious implications that extend much beyond the restricted limits of the medical arena. Thus, it has to be evaluated not only scientifically, but also within a historical, sociological and philosophical context.

In the past, the belief that human races had substantial and clearly delimited biological differences contributed to justify discrimination and was used to oppress and foment injustices, even within the medical context. The concept of race is still loaded with ideology and carries within it relationships of power and domination (8). It is similar to a banana peel: empty, slippery and dangerous.

Thus, our final conclusion is that racial pharmacogenomics fails on grounds of insufficient benefit/cost ratio: it has much to recommend against it and very little scientific 
justification in its favor.

To use racial pharmacogenomics as a palliative measure is tantamount to "embracing ignorance". It erroneously confers persistence and credence to the idea that human races do exist. As pointed out by the sociologist Paul Gilroy (30), such persistence is toxic, contaminating and weakens all society.

The proper course is to wait for the development and establishment of real personalized pharmacogenomics, a true science of discovery. In the next section, I shall examine how soon we can expect that to happen.

\section{Present and future of personalized pharmacogenomics}

At this point I hope to have made clear the necessity to study individual genomes and not races in personalized pharmacogenomics. I propose now to examine the perspectives for the development of this field.

Obviously, the present and future of personalized pharmacogenomics are intimately connected to those of personalized medicine in general. There, we are experiencing a new genomic revolution impelled by massively parallel high-throughput new generation sequencing (31).

Today's DNA sequencers are dozens of thousands times faster than the ones used in the Human Genome Project (32) (Figure 2A). At the same time the price of sequencing a human genome has been dropping exponentially (Figure 2B).

It is possible today to obtain a human genome sequence at Illumina for US\$14,500 (33). The mythic goal of the one thousand dollar genome is in sight and may be achieved as early as 2011 (Figure 2B). As a consequence, incorporation of personal genomes into clinical practice has been achieved (34) and is already becoming commonplace.

Moreover, "next-generation" sequencing is being challenged by even faster and more effective technologies that already loom on the horizon, especially single DNA molecule sequencing and microfluidics.

Recently, the 1000 Genomes Project published its first report, consisting of the whole-genome sequencing of 179 individuals from four populations, high coverage sequencing of two mother-child-father trios and exon-targeted sequencing of 697 individuals (35). Their results indicate that we already have robust protocols for whole genome shotgun sequencing and targeted sequence data and that there are efficient algorithms to detect individual sequence and structural variants in the complete human genome. A survey made by Nature (36) indicates that at least 2700 human genomes have already been sequenced and that
A

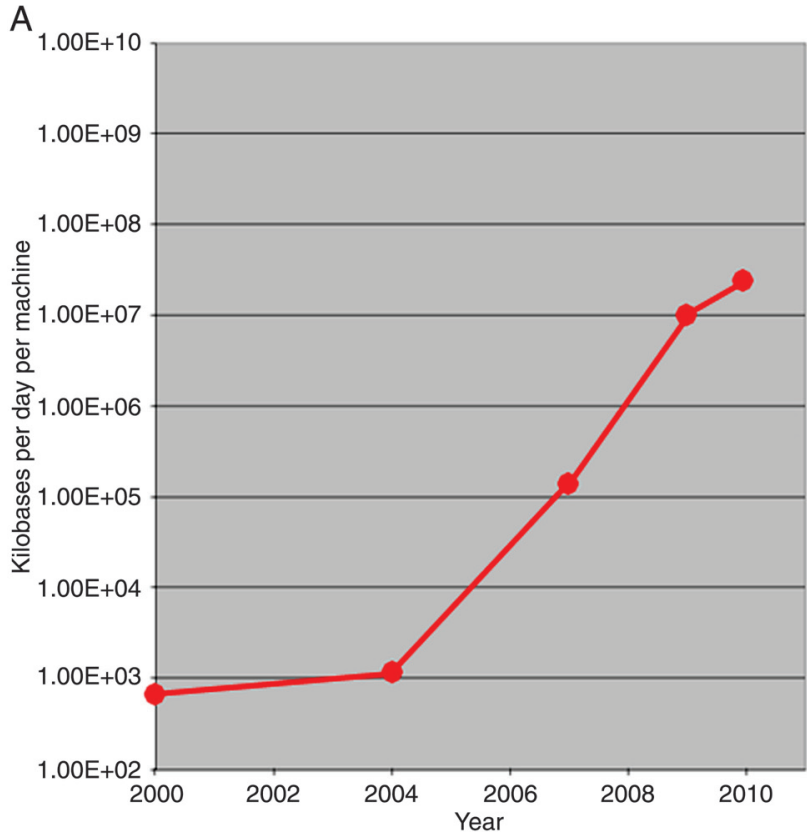

B

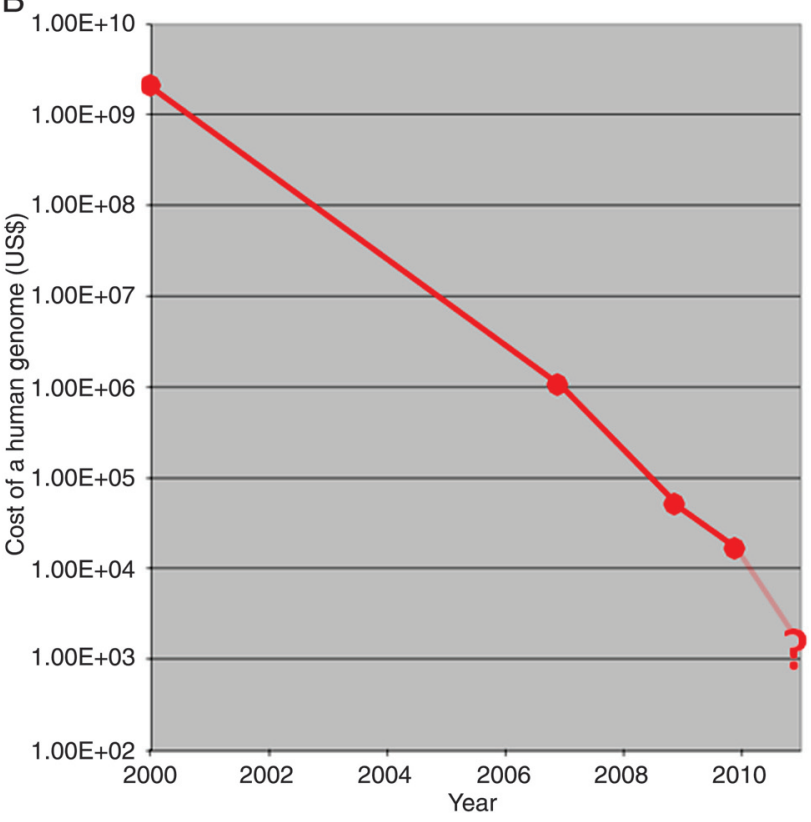

Figure 2. Graphs showing the fantastic progress in the development of rapid and inexpensive human genome next-generation sequencing in the past 10 years. In $A$, the vertical axis, on a logarithmic scale, shows the efficiency of the DNA sequencers, measured in thousands of base pairs (kilobases) per sequencer per day. The graph was plotted using data presented by Venter (Ref. 32). In $B$, the vertical axis, also on a logarithmic scale, shows the price of sequencing a human genome. The graph was plotted using data presented at the following sites (http://www.genengnews.com/gen-news-highlights/illumina-cuts-price-for-individual-genome-sequencingservice-by-more-than-half/81243489/) (http://www.technologyreview.com/biomedicine/24590/?a=f) 
by the end of 2011 that number may reach 30,000 .

However, two hurdles will have to be overcome before personalized pharmacogenomics can really take off. The first is that the prices of sequencing human genomes do not include the necessary bioinformatics interpretation, which is very complex (37).

Fortunately, very recently new strategies have started to appear addressing this problem. One good example involves free software that incorporates simple targeted computational algorithms for do-it-yourself screening of specific genes (38). In this fashion, with user-friendly computer programs it may be possible for physicians and scientists to analyze the genome and to extract the necessary pharmacogenomic information without the need for bioinformatics expertise.

This leads us to the second, more formidable, hurdle of identifying the proper genome content, i.e., new polymorphisms relevant for pharmacogenomics. Up to now, available microarray technology could only study common variants, which confer relatively small increments in risk. In this fashion, only a small proportion of genetic variation in drug handling has been explained so far and the larger part of the pharmacogenomic heritability is still missing (39).

One promising source for uncovering the still unexplained heritability is the investigation of rare, low-

\section{References}

1. Tyson NG. The perimeter of ignorance. http://www.naturalhistorymag.com/universe/211420/the-perimeter-of-ignorance. Accessed January 29, 2011.

2. American Anthropological Association statement on "Race". http://www.aaanet.org/stmts/racepp.htm. Accessed January 29, 2011.

3. http://www.fda.gov/NewsEvents/Newsroom/PressAnnouncements/2005/ucm108445.htm. Accessed January 29, 2011.

4. Jones J, Goodman A. BiDil and the "fact" of genetic blackness. http://www.aaanet.org/press/an/1005/Jones_Goodman.htm. Accessed January 29, 2011.

5. Lewontin RC. The apportionment of human diversity. J Evol Biol 1972; 6: 381-398.

6. Templeton AR. Human races, a genetic and evolutionary perspective. Am Anthropol 1999; 100: 632-650.

7. Evelyn B, Toigo T, Banks D, Pohl D, Gray K, Robins B, et al. Participation of racial/ethnic groups in clinical trials and race-related labeling: a review of new molecular entities approved 1995-1999. J Natl Med Assoc 2001; 93: 18S-24S.

8. Munanga K. Uma abordagem conceitual das noções de raça, racismo, identidade e etnia. In: Brandão AAP (Editor), Cadernos PENESB 5. Niterói: Editora da Universidade Federal Fluminense; 2004. p 16-34.

9. Sturm RA. Molecular genetics of human pigmentation diversity. Hum Mol Genet 2009; 18: R9-R17.

10. Yu N, Chen FC, Ota S, Jorde LB, Pamilo P, Patthy L, et al. Larger genetic differences within Africans than between Africans and Eurasians. Genetics 2002; 161: 269-274. penetrance genetic variants, a class that ranges from lowfrequency polymorphisms (allele frequency $<5 \%$ ) through subpolymorphic variants (frequency $0.1-1.0 \%$ ) to very low frequency or 'private' variants with frequencies of $0.1 \%$ or less (40). If pharmacogenomic variation does indeed depend on the phenotypic effect of many rare variants, the missing heritability of drug responses will then largely be explained and the information will become clinically available.

The wide availability of human whole genome sequencing has removed the technological barriers to the discovery and testing of rare predisposition alleles. The next few months will witness a flood of data that should illuminate the genetics of aspects of pharmacological traits and enhance the potential of personal pharmacogenomics for routine clinical use.

In summary, human clinical genomic knowledge and tools are advancing at enormous speed. Personalized pharmacogenomics should soon become a reality in routine clinical practice.

\section{Acknowledgments}

I am grateful to Fernanda Kehdy for her valuable help in the computations involved in Table 1 and Figure 1.

11. Ng PC, Zhao Q, Levy S, Strausberg RL, Venter JC. Individual genomes instead of race for personalized medicine. Clin Pharmacol Ther 2008; 84: 306-309.

12. Behar DM, Villems R, Soodyall H, Blue-Smith J, Pereira L, Metspalu E, et al. The dawn of human matrilineal diversity. Am J Hum Genet 2008; 82: 1130-1140.

13. Limdi NA, Wadelius M, Cavallari L, Eriksson N, Crawford DC, Lee MT, et al. Warfarin pharmacogenetics: a single VKORC1 polymorphism is predictive of dose across 3 racial groups. Blood 2010; 115: 3827-3834.

14. Cann RL, Stoneking M, Wilson AC. Mitochondrial DNA and human evolution. Nature 1987; 325: 31-36.

15. Frazer KA, Ballinger DG, Cox DR, Hinds DA, Stuve LL, Gibbs RA, et al. A second generation human haplotype map of over 3.1 million SNPs. Nature 2007; 449: 851-861.

16. http://www.sanger.ac.uk/resources/downloads/human/hapmap3.html. Accessed January 29, 2011.

17. Dunn LC, Dobzhansky T. Heredity, race, and society. New York: The New American Library of World Literature; 1946.

18. Rosenberg NA, Pritchard JK, Weber JL, Cann HM, Kidd KK, Zhivotovsky LA, et al. Genetic structure of human populations. Science 2002; 298: 2381-2385.

19. Burchard EG, Ziv E, Coyle N, Gomez SL, Tang H, Karter AJ, et al. The importance of race and ethnic background in biomedical research and clinical practice. $N$ Engl $J$ Med 2003; 348: 1170-1175.

20. Mountain JL, Risch N. Assessing genetic contributions to phenotypic differences among 'racial' and 'ethnic' groups. Nat Genet 2004; 11 (Suppl): S48-S53. 
21. Wade N. Before the dawn, recovering the lost history of our ancestors. New York: Penguin; 2007.

22. Kidd JR, Black FL, Weiss KM, Balazs I, Kidd KK. Studies of three Amerindian populations using nuclear DNA polymorphisms. Hum Biol 1991; 63: 775-794.

23. Serre $D$, Paabo $S$. Evidence for gradients of human genetic diversity within and among continents. Genome Res 2004; 14: 1679-1685.

24. Bastos-Rodrigues L, Pimenta JR, Pena SD. The genetic structure of human populations studied through short insertion-deletion polymorphisms. Ann Hum Genet 2006; 70: 658-665.

25. Nievergelt CM, Libiger O, Schork NJ. Generalized analysis of molecular variance. PLoS Genet 2007; 3: e51.

26. Long JC, Li J, Healy ME. Human DNA sequences: more variation and less race. Am J Phys Anthropol 2009; 139: 23-34.

27. Weiss KM, Long JC. Non-Darwinian estimation, my ancestors, my genes' ancestors. Genome Res 2009; 19: 703710.

28. Foster MW, Sharp RR. Beyond race: towards a wholegenome perspective on human populations and genetic variation. Nat Rev Genet 2004; 5: 790-796.

29. Paabo S. The mosaic that is our genome. Nature 2003; 421 : 409-412.

30. Gilroy P. Against race - imagining political culture beyond the color line. Cambridge: Harvard University Press; 2000.

31. Tucker T, Marra M, Friedman JM. Massively parallel se- quencing: the next big thing in genetic medicine. Am J Hum Genet 2009; 85: 142-154.

32. Venter JC. Multiple personal genomes await. Nature 2010; 464: 676-677.

33. Genetic Engineering \& Biotechnology News. Illumina cuts price for individual genome sequencing service by more than half. http://www.genengnews.com/gen-news-highlights/ illumina-cuts-price-for-individual-genome-sequencingservice-by-more-than-half/81243489. Accessed January 29, 2011.

34. Ashley EA, Butte AJ, Wheeler MT, Chen R, Klein TE, Dewey $\mathrm{FE}$, et al. Clinical assessment incorporating a personal genome. Lancet 2010; 375: 1525-1535.

35. Durbin RM, Abecasis GR, Altshuler DL, Auton A, Brooks LD, Durbin RM, et al. A map of human genome variation from population-scale sequencing. Nature 2010; 467: 10611073.

36. Human genome: Genomes by the thousand. Nature 2010; 467: 1026-1027.

37. Mardis ER. The $\$ 1,000$ genome, the $\$ 100,000$ analysis? Genome Med 2010; 2: 84.

38. Salzberg SL, Pertea M. Do-it-yourself genetic testing. Genome Biol 2010; 11: 404.

39. Manolio TA, Collins FS, Cox NJ, Goldstein DB, Hindorff LA, Hunter DJ, et al. Finding the missing heritability of complex diseases. Nature 2009; 461: 747-753.

40. Bodmer W, Tomlinson I. Rare genetic variants and the risk of cancer. Curr Opin Genet Dev 2010; 20: 262-267. 